Research Paper

\title{
Occurrence and prognosis of lymph node metastases in patients selected for isolated limb perfusion with soft tissue sarcoma
}

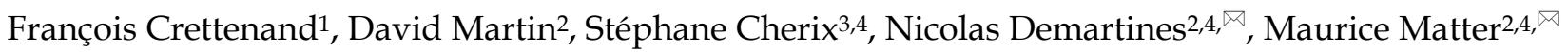 \\ 1. Department of Urology, University Hospital CHUV, Lausanne, Switzerland \\ 2. Department of Visceral Surgery, University Hospital CHUV, Lausanne, Switzerland \\ 3. Department of Orthopaedics and Traumatology, University Hospital CHUV, Lausanne, Switzerland \\ 4. Sarcoma Center, University Hospital CHUV, Lausanne, Switzerland \\ $\triangle$ Corresponding author: Nicolas Demartines, Department of Visceral Surgery, University Hospital CHUV, Rue du Bugnon 46, 1011 Lausanne, Switzerland. \\ E-mail: demartines@chuv.ch \\ (c) Ivyspring International Publisher. This is an open access article distributed under the terms of the Creative Commons Attribution (CC BY-NC) license \\ (https:// creativecommons.org/licenses/by-nc/4.0/). See http://ivyspring.com/terms for full terms and conditions.
}

Received: 2018.02.23; Accepted: 2018.08.03; Published: 2018.09.07

\begin{abstract}
Background and Objectives: Extensive surgery is often required for advanced soft tissue sarcoma (STS) of the limb. In the 1980s, a new approach was developed: isolated limb perfusion (ILP). This study aimed to assess incidence and impact on patient survival based on lymph node metastasis with systematic radical lymphadenectomy during ILP.

Methods: Retrospective study of 57 consecutive patients treated by ILP for limb STS with simultaneous radical lymph node dissection in our tertiary referral center between 1992 and 2015.

Results: Median age was 62 years (19-87) and 30 patients were male (53\%). Lymph node involvement was observed in 13 patients (N1, 23\%), regarded as metastatic spreading in 4 angiosarcomas, 3 epithelioid sarcomas, 2 leiomyosarcomas, 2 undifferentiated sarcomas and 2 synovial sarcomas. For the N0 patient group, median survival was 73.9 months ( $\mathrm{Cl} 95 \%$ 41.9-105.9) compared to 15.1 months $(\mathrm{Cl} 95 \% 7.4-22.6)$ in case of metastatic lymph node $(p=0.002)$. The median disease-free survival was 33,0 months $(\mathrm{Cl} 95 \% 12,5-53.5)$ in N0 group and 8.0 months ( $\mathrm{Cl} 95 \%$ 4.0-11.9) in N1 ( $p=0.006)$.
\end{abstract}

Conclusions: Lymph node metastases of STS patients selected for ILP seemed to have a negative impact on both overall and disease-free survival. Radical lymph node dissection should be included in ILP procedure.

Key words: limb sarcoma, lymph node metastases, incidence, prognosis

\section{Introduction}

Soft tissue sarcomas (STS) are tumours arising from connective tissues, with an incidence of about 5 per $100^{\prime} 000$ [1]. Their prognostic is poor, with an overall 50\% 5-year survival [2,3]. Previously, limb STS were treated by amputation until 1982 when a randomised controlled trial that showed no benefit regarding overall survival (OS) when amputation was compared to limb-sparing resection plus adjuvant radiation therapy [4]. The Isolated Limb Perfusion technique (ILP) was first described in 1958 [5]. The technique has been increasingly used since 1992, when a $90 \%$ remission rate for STS treated by a combination of chemotherapy and Tumour Necrosis
Factor alpha (TNF $\alpha)$ has been demonstrated [6]. More recently, a review has shown that ILP was an acceptable modality for neoadjuvant treatment of limb STS initially regarded as unresectable [7]. Three decades ago, former studies have retained that there was no indication for systematic radical lymph node dissection even in case of locally advanced disease [8-10]. Despite an increasing interest for radical lymph node dissection as part of treatment for sarcomas, there is currently no study on a standardized population in which radical lymph node dissection was performed systematically. 
The study aim was to assess incidence and impact on patient survival of lymph node metastases in limb STS during ILP with systematic radical lymphadenectomy.

\section{Patients and Methods}

\section{Patients}

Retrospective study including all consecutive patients treated by ILP for limb STS with systematic radical lymph node dissection in our tertiary referral centre between 1992 and 2015. Distant metastasis at diagnosis was not an exclusion criterion. Extracted data from medical files included demographics, surgical reports, radiological findings, pathological reports and follow-up. Some patients received neoadjuvant chemotherapy and/or radiotherapy according to local protocols, while others benefited from primary ILP.

\section{Isolated Limb Perfusion (ILP)}

The details of surgical technique have been widely published [6, 11-13]. In brief, the external iliac, the femoral or axillary artery and vein respectively of the diseased limbs are clamped, cannulated and connected to an extracorporeal circuit. In our centre, a radical ilio-inguinal or axillary lymph nodes dissection was systematically performed during vascular dissection to optimize vascular access. To minimize the risk of chemotherapy leakage, the collateral vessels were ligated and a tourniquet applied at the root of the limb. The limb was heated and maintained at a temperature between $38^{\circ}$ and $40^{\circ}$ Celsius. Leaks were assessed with human albumin radiolabelled by Tc99m and a gamma camera placed in precordial position [14]. Once rheological parameters were adjusted and stable, a single dose of Melphalan (Alkeran ${ }^{\circledR}$ - Pro Concepta Zug AG Switzerland) of $13 \mathrm{mg} / 1$ for upper limb and $10 \mathrm{mg} / 1$ for lower limb was perfused. At the same time, from 1 to $4 \mathrm{mg}$ of recombinant Tumour Necrosis Factor alpha (Beromun ${ }^{\circledR}$ Boehringer-Ingelheim $\mathrm{GmbH}$ Ingelheim Germany) was added [15]. After 60 minutes of perfusion, the limb was rinsed with up to 4 litres of saline solution then the systemic circulation restored.

\section{Radical lymph node dissection}

Pelvic radical lymph node dissections (RNLD) included external iliac and obturator lymph nodes. Axillary RLND included level I - III dissection. In this study, RNLD was performed regardless of clinical or radiological lymph node status.

\section{Tumour and staging}

The histologic grading system used was the FNCLC (Fédération National des Centres de Lutte contre le Cancer) $[16,17]$.

\section{Statistical analysis}

Quantitative variables were presented as mean (standard deviation) and compared with Mann-Whitney $U$ test. Qualitative variables were presented as frequencies (percentage) and compared with Pearson's chi-square or Fisher's exact test as appropriate. The OS was calculated from the date of ILP until patient's death or last follow-up. The disease-free survival was calculated from ILP until local recurrence or metastases documented by MRI or CT-scan (regular follow-up every 3 months with clinical work up and imaging). Patients with initial distant metastases were excluded from the disease-free survival analysis. The survival curves were drawn according to Kaplan-Meier's method with a Log Rank test (Mantel-Cox) to compare groups. A $p$ value $\leq 0.05$ was considered statistically significant. All statistical analyses were performed using SPSS 23.0 software (SPSS Inc., Chicago, IL).

\section{Ethics}

The study was conducted according to the Declaration of Helsinki principles of good clinical practice and approved by the Local Ethics' Committee (Protocol number 392/11).

\section{Results}

Fifty-seven patients had ILP with radical lymph node dissection for limb STS. Four patients had a second ILP and only the first intervention was considered. Median age was 62 years (range 19-87, SD $17.3)$ and 30 patients were male (53\%). Seven patients had upper limb (12\%) and 50 lower limb (88\%) STS. Only 4 patients had synchronous distant metastases (3 with lung and 1 with liver metastases). The various histological types of sarcomas are described according to lymph node involvement in Table 1.

Table 1. Histology of sarcomas and lymph node involvement

\begin{tabular}{llll}
\hline & $\begin{array}{l}\text { N-positive } \\
\mathrm{n}=13\end{array}$ & $\begin{array}{l}\text { N-negative } \\
\mathrm{n}=44\end{array}$ & $\begin{array}{l}\text { Total } \\
\mathrm{n}=57\end{array}$ \\
\hline Angiosarcomas & 4 & 3 & 7 \\
Epithelioid sarcomas & 3 & 1 & 4 \\
Leiomyosarcomas & 2 & 3 & 5 \\
Synovial sarcomas & 2 & 2 & 4 \\
Undifferentiated sarcomas & 2 & 8 & 10 \\
Liposarcomas & 0 & 13 & 13 \\
Pleiomorphic sarcomas & 0 & 5 & 5 \\
Neurofibrosarcoma & 0 & 2 & 2 \\
Clear cell sarcoma & 0 & 1 & 1 \\
Osteosarcoma & 0 & 1 & 1 \\
Desmoid tumour & 0 & 1 & 1 \\
Unclassified subtypes & 0 & 4 & 4 \\
\hline
\end{tabular}

Mean number of resected lymph node was 9.5 (range 1-41, SD 7.2). Lymph node metastatic disease 
was observed in 13 patients (N1, 23\%): 4 angiosarcomas, 3 epithelioid sarcomas, 2 leiomyosarcomas, 2 undifferentiated sarcomas and 2 synovial sarcomas. No statistically significant correlation was found between tumour size, location and development of nodal metastasis (Table 2). In terms of tumour grades, patients with nodal involvement (N1) had significantly more grade 3 compared to N0 group ( $69 \%$ vs. $32 \%$, p $=0.019)$.

Table 2. Oncological properties of sarcomas and lymph node involvement

\begin{tabular}{|c|c|c|c|}
\hline & $\begin{array}{l}\text { N-positive } \\
n=13\end{array}$ & $\begin{array}{l}\mathrm{N} \text {-negative } \\
\mathrm{n}=44\end{array}$ & P-value \\
\hline Mean tumour size in $\mathrm{cm}$ (SD) & $10.6(5.1)$ & $10.1(6.1)$ & 0.627 \\
\hline \multicolumn{4}{|l|}{ Tumour grade, n (\%) } \\
\hline G1 & 0 & $3(7)$ & 0.453 \\
\hline G2 & $4(31)$ & $27(61)$ & 0.052 \\
\hline G3 & $9(69)$ & $14(32)$ & 0.019 \\
\hline \multicolumn{4}{|l|}{ Tumour localisation, $\mathrm{n}(\%)$} \\
\hline Upper limb & & & 0.643 \\
\hline Proximal & $1(8)$ & $4(9)$ & \\
\hline Distal & $1(8)$ & $2(5)$ & \\
\hline Lower limb & & & 0.161 \\
\hline Proximal & $8(61)$ & $19(43)$ & \\
\hline Distal & $3(23)$ & $19(43)$ & \\
\hline
\end{tabular}

For the N0 patient group, median survival was 73.9 months (CI $95 \%$ 41.9-105.9) compared to 15.1 months (CI 95 \% 7.4-22.6) in case of metastatic lymph node(s) (N1) ( $\mathrm{p}=0.002$, Fig. 1). Median disease-free survival was 33,0 months (CI 95\% 12,5-53.5) in N0 group and 8.0 months (CI 95\% 4.0-11.9) in N1 $(p=0.006$, Fig. 2). There was no significant overall or disease-free survival between histological types, although the small number of cases limited the analysis.

\section{Discussion}

This present study is one of the few that adopted systematic lymph node resection during ILP. Lymph node involvement was found in $23 \%$ of patients, which is slightly higher than what was usually published: nodal metastasis rate in the literature varies between $1,3 \%$ and $24,7 \%$ according to studied populations and histological subtypes [9, 10, 18-21]. However, a recently published retrospective study of patients with STS showed that nodal metastasis rates for STS histologic subtypes were higher than previously reported. This discrepancy could be explained by the absence of standardized lymph node evaluation among patients who underwent surgery for STS. According to other studies, RLND was performed only when lymph drainage area was included in resection's margins [9, 21]. Moreover, other authors considered RLND only in patients with preoperative evidence of lymph nodes metastases [18].
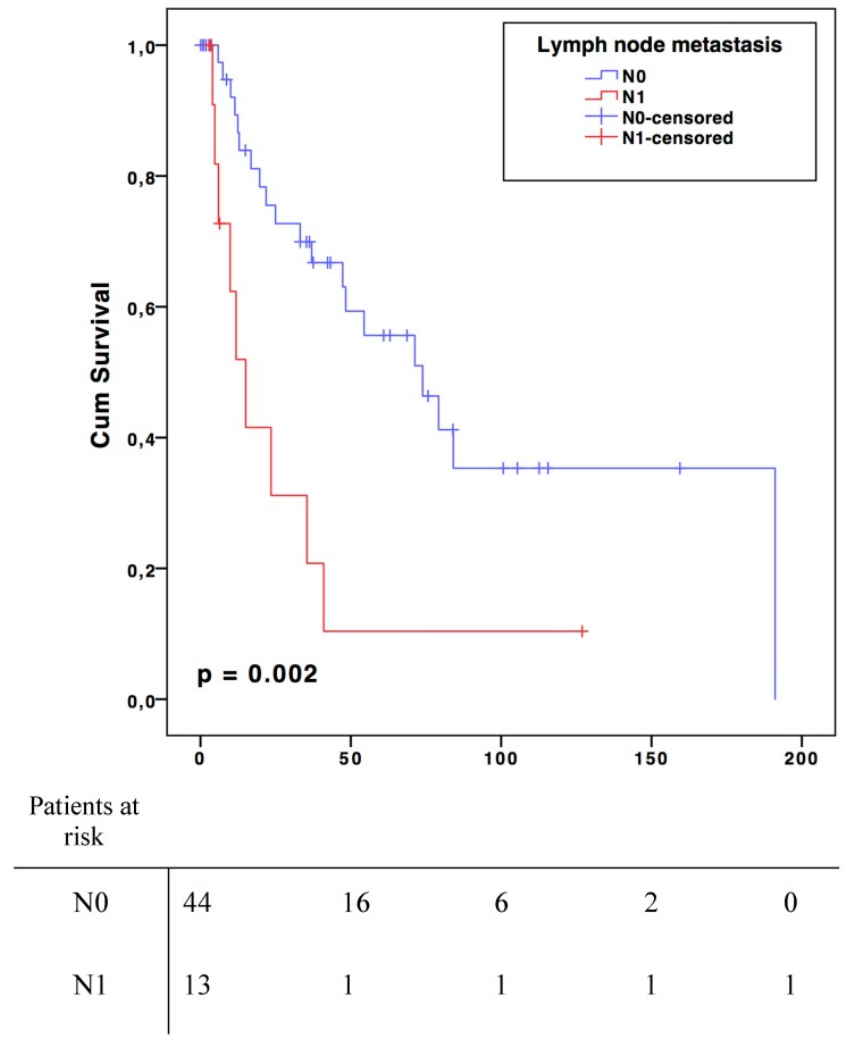

Figure 1. Overall Survival

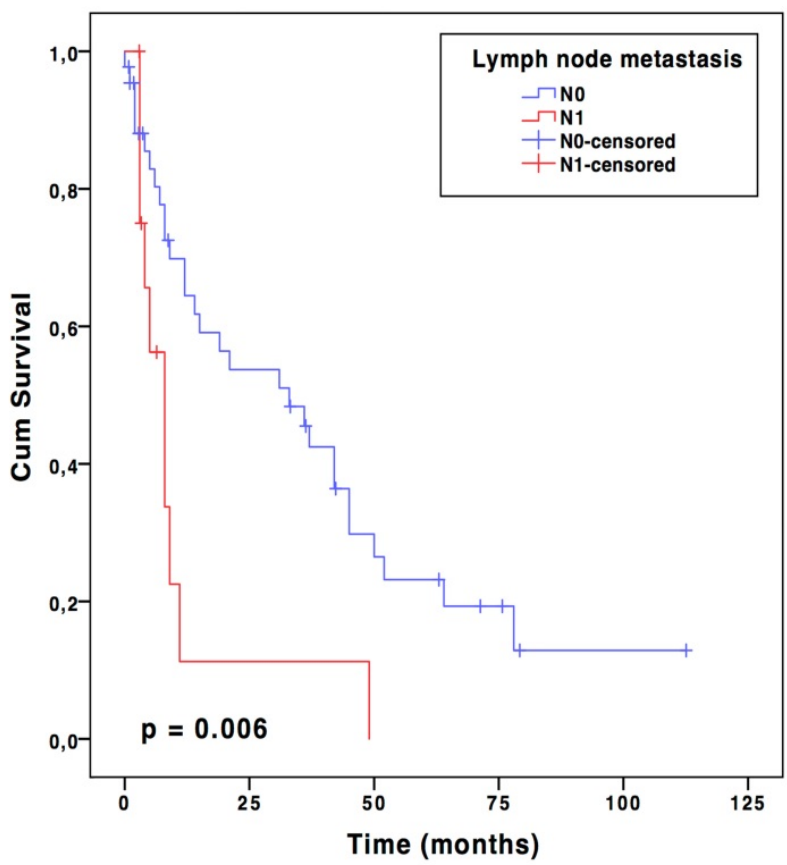

Patients at risk

\begin{tabular}{l|lllll} 
N0 & 41 & 20 & 9 & 4 & 1 \\
N1 & 12 & 1 & 0 & 0 & 0
\end{tabular}

Figure 2. Disease-free survival 
In the present study, lymph node metastases of STS seemed to have a negative impact on both OS and disease-free survival. There was a significant decline of 59-months in overall survival for patients with lymph node involvement. There was also a 37-month difference in disease-free survival. Lymph node involvement appeared to be a factor that clinically reflects the biological aggressiveness of the tumour $[18,21]$. It is interesting to note that despite the negative impact of lymph node involvement, the American Joint Committee on Cancer (AJCC) revised its impact on survival. Indeed, in the two last cancer staging manual editions (2010 and 2016) N1 sarcomas were classified as stage III and no longer as stage IV compared to the 2002 version [22]. Stage IV now includes only distant metastatic patients. Lymph node involvement alone is not as aggravating as distant metastases in term of prognosis [21]. The OS benefit offered when the tumour did not reached the lymph nodes in addition together with potentially curative aspect of RLND are major arguments in favour of systematic lymph node dissection during ILP [23]. Moreover, the benefits of this treatment appear to be higher than its potential morbidities (lymphedema, lymphocele, infection and hematoma) [24]. The results from the present study suggest that lymph node dissection should be done systematically at least when an ILP procedure is performed. However, this cannot be claimed for sarcomas in general.

Given the potential benefit of recognizing and removing occult lymph node involvement, some centres proposed to search sentinel node in patients with STS. Recently, a prospective study demonstrated the safety and accuracy of sentinel lymph node biopsy compared to PET-CT to identify therapy-changing nodal disease [25]. However, a meta-analysis recommended sentinel lymph node biopsy prior to radical lymphadenectomy only in sarcoma subtypes with a high risk of lymph node involvement [20]. A retrospective study of an American National Cancer Data Base identified that nodal metastasis rates were highest among patients with rhabdomyosarcoma $(32.1 \%)$, angiosarcoma (24.1\%), clear cell sarcoma $(27.7 \%)$, and epithelioid sarcoma $(31.8 \%)$, which is similar to this series as well as another retrospective study $[9,26]$. From the anatomical point of view, it has been shown that proximal-type sarcomas have significantly more aggressive clinicopathological features, particularly lymph node metastasis [27]. Regarding tumour grade, high grade sarcomas tend to have lymph node metastases $(69 \%)$, as previously reported by some authors in retrospective studies [26, 28]. The fact that some STS subtypes are at high risk for nodal involvement should be included in treatment planning. For sarcomas with potentially high lymph node involvement risk, an active detection of lymph node metastases may be evaluated preoperatively (sentinel lymph node biopsy, MRI/CT or PET-CT) [29]. Unfortunately, given the small number of patients in the present series, conclusions and recommendations cannot be drawn for "high-risk" histologies. In the absence of clear recommendations, systematic lymph node dissection during ILP is performed in our academic center. However, according to results of this series and other retrospective studies, it could be considered to perform a RLND for high risk sarcomas (rhabdomyosarcoma, angiosarcoma, clear cell sarcoma, epithelioid sarcoma, synovial sarcoma) and not only during ILP procedures [26, 29].

Conventional imaging modalities (CT-scan and MRI) show a poor sensitivity (small nodal metastases) and specificity (inflammation) for the detection of lymph node metastases. On contrary, ${ }^{18}$ F-FDG-PET has proven to be a promising imaging modality for detecting primary tumours, lymph node metastases and bone involvement of sarcomas, with better sensitivity than conventional methods including ultrasound and bone scintigraphy [30]. Another previously published study estimated a positive predictive value for lymph node metastasis of $27 \%$ [31]. In addition, ${ }^{18}$ F-FDG-PET can also be used in the evaluation and monitoring of tumour response to therapy. Nevertheless, a discrepancy in $42.9 \%$ of cases between the pathological evaluation and that obtained by PET has been described [32].

The main limitation of the present study is its retrospective nature and a relatively small number of patients that prevented multivariate analysis. Furthermore, a wide range of histological subtypes was included. On the other hand, sarcoma is an orphan disease among adult and paediatric populations. Currently, the lack of clear recommendations regarding lymphadenectomy in STS probably leads to an underestimation of lymph node metastases rate and its impact on patient's mortality. Moreover, the same limit applies to neoadjuvant treatments, because there is currently no clear accepted protocol. More research is needed to make evidence-based recommendations in favour of systematic radical lymph node dissection as part of the surgical treatment. The definitive role of RLND in ILP's for limb sarcoma patients' needs more studies.

In conclusion, lymph node metastases of soft tissue sarcoma patients selected for regional therapy (ILP) seemed to have a negative impact on both overall and disease-free survival. Systematic radical lymph node dissection should be included in ILP procedures to have more precise diagnosis with possible positive impact on outcome. 


\section{Note}

This paper was presented in parts as a poster to the Annual Meeting of the Swiss Society of Visceral Surgery (SSCV), September 2017, Lausanne, Switzerland.

\section{Competing Interests}

The authors have declared that no competing interest exists.

\section{References}

1. Burningham Z, Hashibe M, Spector L, Schiffman JD. The epidemiology of sarcoma. Clinical sarcoma research. 2012; 2: 14

2. Levi F, La Vecchia C, Randimbison L, Te VC. Descriptive epidemiology of soft tissue sarcomas in Vaud, Switzerland. European journal of cancer (Oxford, England : 1990). 1999; 35: 1711-6.

3. Pollock RE, Karnell LH, Menck HR, Winchester DP. The National Cancer Data Base report on soft tissue sarcoma. Cancer. 1996; 78: 2247-57.

4. Rosenberg SA, Tepper J, Glatstein E, Costa J, Baker A, Brennan M, et al. The treatment of soft-tissue sarcomas of the extremities: prospective randomized evaluations of (1) limb-sparing surgery plus radiation therapy compared with amputation and (2) the role of adjuvant chemotherapy. Annals of surgery. 1982; 196: 305-15.

5. Creech O, Jr., Krementz ET, Ryan RF, Winblad JN. Chemotherapy of cancer: regional perfusion utilizing an extracorporeal circuit. Annals of surgery. 1958; 148: 616-32.

6. Lienard D, Ewalenko P, Delmotte JJ, Renard N, Lejeune FJ. High-dose recombinant tumor necrosis factor alpha in combination with interferon gamma and melphalan in isolation perfusion of the limbs for melanoma and sarcoma. Journal of clinical oncology : official journal of the American Society of Clinical Oncology. 1992; 10: 52-60.

7. Jakob J, Tunn PU, Hayes AJ, Pilz LR, Nowak K, Hohenberger P. Oncological outcome of primary non-metastatic soft tissue sarcoma treated by neoadjuvant isolated limb perfusion and tumor resection. Journal of surgical oncology. 2014; 109: 786-90.

8. Weingrad DN, Rosenberg SA. Early lymphatic spread of osteogenic and soft-tissue sarcomas. Surgery. 1978; 84: 231-40.

9. Mazeron JJ, Suit HD. Lymph nodes as sites of metastases from sarcomas of soft tissue. Cancer. 1987; 60: 1800-8.

10. Collin C, Godbold J, Hajdu S, Brennan M. Localized extremity soft tissue sarcoma: an analysis of factors affecting survival. Journal of clinical oncology : official journal of the American Society of Clinical Oncology. 1987; 5: 601-12.

11. Eggermont AM, Schraffordt Koops H, Klausner JM, Kroon BB, Schlag PM, Lienard D, et al. Isolated limb perfusion with tumor necrosis factor and melphalan for limb salvage in 186 patients with locally advanced soft tissue extremity sarcomas. The cumulative multicenter European experience. Annals of surgery. 1996; 224: 756-64; discussion 64-5.

12. Eggermont AM, de Wilt JH, ten Hagen TL. Current uses of isolated limb perfusion in the clinic and a model system for new strategies. The Lancet Oncology. 2003; 4: 429-37.

13. Matter M DA, Gay B. Induction treatment in sarcomas. In: Aigner KR, Stephens FO eds Induction chemotherapy Second edition Springer Heidelberg 2016. pp391-436.

14. Hoekstra HJNaujocks TSchraffordt Koops $\mathrm{H}$ et al. Continuous leakage monitoring during hyperthermic isolated regional perfusion of the lower limb: techniques and results. Region Cancer Treat. 1992;4301- 304

15. Lejeune FJ, Lienard D, Matter M, Ruegg C. Efficiency of recombinant human TNF in human cancer therapy. Cancer immunity. 2006; $6: 6$.

16. Trojani M, Contesso G, Coindre JM, Rouesse J, Bui NB, de Mascarel A, et al. Soft-tissue sarcomas of adults; study of pathological prognostic variables and definition of a histopathological grading system. International journal of cancer. 1984; 33: 37-42.

17. Guillou L, Coindre JM, Bonichon F, Nguyen BB, Terrier P, Collin F, et al Comparative study of the National Cancer Institute and French Federation of Cancer Centers Sarcoma Group grading systems in a population of 410 adult patients with soft tissue sarcoma. Journal of clinical oncology : official journal of the American Society of Clinical Oncology. 1997; 15: 350-62.

18. Ruka W, Emrich LJ, Driscoll DL, Karakousis CP. Prognostic significance of lymph node metastasis and bone, major vessel, or nerve involvement in adults with high-grade soft tissue sarcomas. Cancer. 1988; 62: 999-1006.

19. Fong Y, Coit DG, Woodruff JM, Brennan MF. Lymph node metastasis from soft tissue sarcoma in adults. Analysis of data from a prospective database of 1772 sarcoma patients. Annals of surgery. 1993; 217: 72-7.

20. Wright S, Armeson K, Hill EG, Streck C, Leddy L, Cole D, et al. The role of sentinel lymph node biopsy in select sarcoma patients: a meta-analysis. American journal of surgery. 2012; 204: 428-33.

21. Behranwala KA, A'Hern R, Omar AM, Thomas JM. Prognosis of lymph node metastasis in soft tissue sarcoma. Annals of surgical oncology. 2004; 11: 714-9.
22. AJCC Cancer Staging Manuel Seventh edition. Edge SB, Byrd DR, Compton C, Fritz AG, Greene FL, 22. Trotti III A eds. Soft tissue sarcoma, pp 291-298. Springer New York, Dordrecht, Heidelberg, London 2010.

23. Ferguson PC, Deheshi BM, Chung P, Catton CN, O'Sullivan B, Gupta A, et al. Soft tissue sarcoma presenting with metastatic disease: outcome with primary surgical resection. Cancer. 2011; 117: 372-9.

24. de Vries M, Vonkeman WG, van Ginkel RJ, Hoekstra HJ. Morbidity after inguinal sentinel lymph node biopsy and completion lymph node dissection in patients with cutaneous melanoma. European journal of surgical oncology : the journal of the European Society of Surgical Oncology and the British Association of Surgical Oncology. 2006; 32: 785-9.

25. Wagner LM, Kremer N, Gelfand MJ, Sharp SE, Turpin BK, Nagarajan R, et al. Detection of lymph node metastases in pediatric and adolescent/young adult sarcoma: Sentinel lymph node biopsy versus fludeoxyglucose positron emission tomography imaging-A prospective trial. Cancer. 2017; 123: 155-60.

26. Sherman KL, Kinnier CV, Farina DA, Wayne JD, Laskin WB, Agulnik M, et al. Examination of national lymph node evaluation practices for adult extremity soft tissue sarcoma. Journal of surgical oncology. 2014; 110: 682-8.

27. Asano N, Yoshida A, Ogura K, Kobayashi E, Susa M, Morioka H, et al. Prognostic Value of Relevant Clinicopathologic Variables in Epithelioid Sarcoma: A Multi-Institutional Retrospective Study of 44 Patients. Annals of surgical oncology. 2015; 22: 2624-32.

28. Bianchi G, Sambri A, Righi A, Dei Tos AP, Picci P, Donati D. Histology and grading are important prognostic factors in synovial sarcoma. European journal of surgical oncology : the journal of the European Society of Surgical Oncology and the British Association of Surgical Oncology. 2017; 43: 1733-9.

29. Noebauer-Huhmann IM, Weber MA, Lalam RK, Trattnig S, Bohndorf K, Vanhoenacker F, et al. Soft Tissue Tumors in Adults: ESSR-Approved Guidelines for Diagnostic Imaging. Seminars in musculoskeletal radiology. 2015; 19: e1.

30. Benz MR, Tchekmedyian N, Eilber FC, Federman N, Czernin J, Tap WD. Utilization of positron emission tomography in the management of patients with sarcoma. Current opinion in oncology. 2009; 21: 345-51.

31. Fuglo HM, Jorgensen SM, Loft A, Hovgaard D, Petersen MM. The diagnostic and prognostic value of (1)(8)F-FDG PET/CT in the initial assessment of high-grade bone and soft tissue sarcoma. A retrospective study of 89 patients. European journal of nuclear medicine and molecular imaging. 2012; 39: 1416-24

32. Iagaru A, Masamed R, Chawla SP, Menendez LR, Fedenko A, Conti PS. F-18 FDG PET and PET/CT evaluation of response to chemotherapy in bone and oft tissue sarcomas. Clinical nuclear medicine. 2008; 33: 8-13. 